\title{
On the Cognitive Interference Channel with Causal Unidirectional Destination Cooperation
}

\author{
Fernando Reátegui, Hossein Peyvandi, Muhammad Ali Imran and Rahim Tafazolli
}

\begin{abstract}
In previous works the cognitive interference channel with unidirectional destination cooperation has been studied. In this model the cognitive receiver acts as a relay of the primary user's message and its operation is assumed to be strictly causal. In this paper we study the same channel model with a causal rather than a strictly causal relay, i.e. the relay's transmit symbol depends not only on its past but also on its current received symbol. We propose an outer bound for the discrete memoryless channel which is later used to compute an outer bound for the Gaussian channel. We also propose an achievable scheme based on instantaneous amplify-and-forward relaying that meets the outer bound in the very strong interference regime.
\end{abstract}

Index Terms-Cognitive Interference Channel, Causal Unidirectional Destination Cooperation.

\section{INTRODUCTION}

$\mathbf{T}$ HE cognitive interference channel (CIC) [1] is a model for unidirectional cooperation at the transmitters. In this model two transmitter-receiver pairs interfere with each other and one transmitter has non-causal knowledge of the message being sent by the other transmitter. This knowledge, referred here as cognition, is appropriately utilised by the corresponding encoder in order to apply sophisticated techniques with the aim of increasing the overall rate region. This model has been inspired by applications in mobile communications and sensor networks. In the former, a cognitive radio has special capabilities such that he can utilise the same spectrum as the licensed user [2]. In the latter, two sensors have different sensing capabilities that originate a degraded structure of the messages that need to be communicated. This structure permits the transmitters to cooperate [3].

On the other hand, it has been shown in the literature that cooperative communications can improve the achievable rate regions of several channel configurations. Recently in [4] and [5], the CIC is adapted to have unidirectional cooperation at the receivers (CIC-UDC). The cognitive receiver acts as a strictly causal relay and helps to convey the primary user's message. In this paper we extend this setting and assume that the relay operation is causal rather than strictly causal, i.e., the relay's transmit symbol depends not only on its past received symbols, but also on its current received symbol. We name this model the CIC with causal unidirectional destination cooperation (CIC-CUDC). This is a good model for studying amplify-and-forward (AF) type relaying if the overall delay spread including the path through the relay is much smaller than the inverse of the bandwidth. We provide

F. Reátegui, M. A. Imran and R. Tafazolli are with the Centre of Communication Systems Research (CCSR), University of Surrey, Guildford, Surrey, GU2 7XH UK e-mail: f.reategui@surrey.ac.uk.

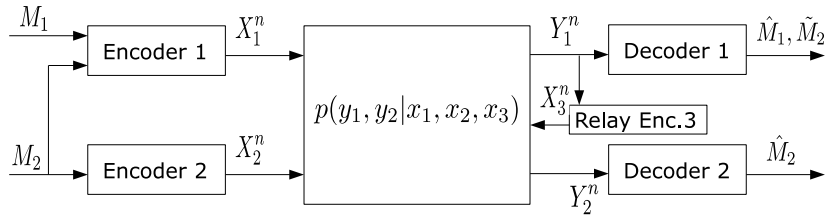

Fig. 1. The discrete memoryless CIC with causal unidirectional destination cooperation. The cognitive transmitter is denoted with the subscript 1 . The cognitive receiver acts as a relay.

an outer bound for the discrete memoryless (DM) channel. For the Gaussian channel we provide an achievable scheme that utilises instantaneous $\mathrm{AF}$ at the relay. We show that this simple encoding scheme is capacity achieving when the model operates in the very strong interference (VSI) regime. The rest of the paper is organised as follows. The notations and definitions are in Section II. An outer bound to the capacity region of the discrete memoryless CIC-UDC is provided in Section III. We establish the capacity region for the Gaussian channel in Section IV. We conclude the paper in Section V.

\section{Notations AND DEFINITIONS}

\section{A. Notations}

The notation of [6] is utilised throughout the paper. Lower case letters (e.g. $x, y$ ) are used to denote values of random variables. Upper case letters (e.g. $X, Y$ ) denote random variables. A sequence of random variables $\left(X_{i}, \ldots X_{j}\right)$ is denoted by $X_{i}^{j}$, for $1 \leq i \leq j$. When $i=1$ the subscript is dropped $X^{j}=\left(X_{1}, \ldots, X_{j}\right)$. The probability mass function (pmf) of a random variable (RV) $X$ is denoted by $p_{X}(x)$. We often drop the subscript when the pmf is understood from the context, e.g. $p(x)$. The entropy (differential entropy) of a $\mathrm{RV}$ and mutual information between two RVs are indicated by $H(\cdot)(h(\cdot))$ and $I(\cdot ; \cdot)$ respectively. The capacity function is defined as $\mathcal{C}(x)=1 / 2 \log (1+x)$ where the logarithm is to the base 2 .

\section{B. Definitions}

Definition 1. The discrete memoryless CIC-CUDC consists of three finite input sets $\mathcal{X}_{1}, \mathcal{X}_{2}, \mathcal{X}_{3}$, two finite output sets $\mathcal{Y}_{1}$, $\mathcal{Y}_{2}$, and a probability transition function $p\left(y_{1}, y_{2} \mid x_{1}, x_{2}, x_{3}\right)$. It is depicted in Fig. 1.

Definition 2. A $\left(2^{n R_{1}}, 2^{n R_{2}}, n\right)$ code for the discrete memoryless CIC-CUDC consists of a pair of uniformly distributed messages $M_{1} \in\left[1: 2^{n R_{1}}\right]$ and $M_{2} \in\left[1: 2^{n R_{2}}\right]$, two encoding functions at the transmitters $X_{1}^{n}=f_{1}\left(M_{1}, M_{2}\right)$, $X_{2}^{n}=f_{2}\left(M_{2}\right)$, an encoding function at the relay $X_{3 i}=$ 


$$
\begin{aligned}
& h_{11}^{2}\left(1-\rho^{2}\right) P_{1} \leq P_{1}(\left.h_{21}^{2}\left(1-\rho^{2}\right)+h_{11}^{2} h_{23}^{2}\left(1-\rho^{2}\right) \alpha^{2}+2 h_{11} h_{21} h_{23}\left(1-\rho^{2}\right) \alpha\right) /\left(1+h_{23}^{2} \alpha^{2}\right), \\
& h_{21}^{2} P_{1}+2 h_{23} \alpha\left(h_{11} h_{21} P_{1}+h_{12} h_{22} P_{2}\right)+2 h_{21} h_{22} \rho \sqrt{P_{1} P_{2}} \\
&+2 h_{23} \alpha \rho \sqrt{P_{1} P_{2}}\left(h_{12} h_{21}+h_{11} h_{22}\right)+h_{22}^{2} P_{2} \leq h_{11}^{2} P_{1}+2 h_{11} h_{12} \rho \sqrt{P_{1} P_{2}}+h_{12}^{2} P_{2}
\end{aligned}
$$

$f_{3 i}\left(Y_{1}^{i}\right)$ and two decoding functions $\hat{M}_{t}=g_{t}\left(Y_{t}^{n}\right)$, for $t=1,2$. The average probability of error is defined as $P_{e}^{(n)}=P\left(\bigcup_{t}\left\{\hat{M}_{t} \neq M_{t}\right\} \bigcup\left\{\tilde{M}_{2} \neq M_{2}\right\}\right)$. A rate pair $\left(R_{1}, R_{2}\right)$ is said to be achievable if there exists a sequence of $\left(2^{n R_{1}}, 2^{n R_{2}}, n\right)$ codes such that $\lim _{n \rightarrow \infty} P_{e}^{(n)}=0$. The capacity region of the discrete memoryless CIC-CUDC is the closure of the set of all achievable rate regions. We assume that the channel is memoryless, i.e., $\left(X_{1}^{i-1}, X_{2}^{i-1}, Y_{1}^{i-1}\right) \rightarrow$ $\left(X_{1 i}, X_{2 i}\right) \rightarrow Y_{1 i}$, and $\left(X_{1}^{i-1}, X_{2}^{i-1}, X_{3}^{i-1}, Y_{1}^{i-1}, Y_{2}^{i-1}\right) \rightarrow$ $\left(X_{1 i}, X_{2 i}, X_{3 i}, Y_{1 i}\right) \rightarrow Y_{2 i}$, form Markov chains.

\section{THE CIC WITH CAUSAL UNIDIRECTIONAL DESTINATION COOPERATION}

We first propose an outer bound for the discrete memoryless CIC-CUDC.

Theorem 1. The capacity region of the discrete memoryless CIC with causal unidirectional cooperation at the receivers is contained in the set of rate pairs $\left(R_{1}, R_{2}\right)$ such that

$$
\begin{aligned}
R_{1} & \leq I\left(X_{1} ; Y_{1}, X_{3} \mid X_{2}\right), \\
R_{1}+R_{2} & \leq I\left(X_{1}, X_{2} ; Y_{1}, X_{3}\right), \\
R_{1}+R_{2} & \leq I\left(X_{1}, X_{2} ; Y_{1}\right)+I\left(X_{1}, X_{2} ; Y_{2} \mid Y_{1}, X_{3}\right),
\end{aligned}
$$

for some $p\left(x_{1}, x_{2}\right) p\left(x_{3} \mid x_{1}, x_{2}, y_{1}\right)$.

Proof. See Appendix A.

We treat the Gaussian channel in the next section.

\section{The Gaussian CIC With CAUSAl UNidiRectional DESTINATION COOPERATION}

In the Gaussian channel the relay node (receiver 1) is equipped with one antenna for reception and another one for transmission. The antennas are isolated, therefore they do not interfere with each other. The channel model is described by the following set of equations:

$$
\begin{aligned}
& Y_{1}=h_{11} X_{1}+h_{12} X_{2}+Z_{1}, \\
& Y_{2}=h_{21} X_{1}+h_{22} X_{2}+h_{23} X_{3}+Z_{2},
\end{aligned}
$$

where $h_{k l}$ is the channel coefficient from transmitter $l$ to receiver $k$ for $k=1,2$ and $l=1,2,3$. The signal transmitted at receiver 1 is denoted by $X_{3} . Z_{k} \sim \mathcal{N}(0,1)$ are zero-mean unit-variance Gaussian noise. The average power constraints at the three transmitters are $P_{1}, P_{2}$ and $P_{3}$ respectively.
A. The Gaussian CIC with causal unidirectional destination cooperation in very strong interference

Definition 3. The relay in the Gaussian CIC-CUDC operates on instantaneous amplify-and-forward relaying, $X_{3}=\alpha Y_{1}$, where $\alpha$ is the amplification factor.

Definition 4. A Gaussian CIC with causal unidirectional destination cooperation is said to be in the very strong interference regime if (3) holds, where $\rho \in[0,1]$ is the correlation coefficient between $X_{1}$ and $X_{2}$.

Theorem 2. The capacity region of the Gaussian CIC with causal unidirectional destination cooperation in very strong interference is given by the set of $\left(R_{1}, R_{2}\right)$ such that

$$
\begin{aligned}
R_{1} & \leq \mathcal{C}\left(h_{11}^{2}\left(1-\rho^{2}\right) P_{1}\right), \\
R_{1}+R_{2} & \leq \mathcal{C}\left(h_{11}^{2} P_{1}+2 h_{11} h_{12} \rho \sqrt{P_{1} P_{2}}+h_{12}^{2} P_{2}\right) .
\end{aligned}
$$

Proof. Achievability: Achievability follows by instantaneous $\mathrm{AF}$ at the relay, i.e., $X_{3}=\alpha Y_{1}$. Substituting this in (2) we obtain:

$$
\begin{aligned}
& Y_{1}= h_{11} X_{1}+h_{12} X_{2}+Z_{1}, \\
& Y_{2}=\left(h_{21}+\alpha h_{11} h_{23}\right) X_{1}+\left(h_{22}+\alpha h_{12} h_{23}\right) X_{2} \\
& \quad+\alpha h_{23} Z_{1}+Z_{2} .
\end{aligned}
$$

As we are studying the very strong interference regime, the equivalent CIC in (5) is in VSI, i.e., the conditions in [7] hold:

$$
\begin{aligned}
& I\left(X_{1} ; Y_{1} \mid X_{2}\right) \leq I\left(X_{1} ; Y_{2} \mid X_{2}\right), \\
& I\left(X_{1}, X_{2} ; Y_{2}\right) \leq I\left(X_{1}, X_{2} ; Y_{1}\right) .
\end{aligned}
$$

As both receivers decode both messages, from the left hand side (LHS) of (6a) the bound on $R_{1}$ in (4a) can be obtained. The LHS of (6b) depends on the amplification factor $\alpha$. The value of $\alpha$ that maximizes the sum rate (LHS of (6b)) makes $I\left(X_{1}, X_{2} ; Y_{2}\right) \geq I\left(X_{1}, X_{2} ; Y_{1}\right)$ for all values of channel gains, namely we are out of the very strong interference regime. This can be observed in Fig. 2. In the figure, when $\alpha=\alpha_{o p t}$ the LHS of (6b) $\left(L H S_{2}\right)$ is at its maximum. The value of $\alpha$ for which the LHS and the right hand side (RHS) of (6b) equalize is denoted by $\alpha_{e q}$.

Therefore we need to compute the value of $\alpha$ such that we attain the maximum sum-rate without leaving the very strong interference regime. The value of $\alpha$ such that the LHS and RHS of (6b) are equal is computed to be $\alpha_{e q}=\frac{N}{D}$ where $N=h_{11}^{2} P_{1}+2 h_{11} h_{12} \rho \sqrt{P_{1} P_{2}}+h_{12}^{2} P_{2}-\left(h_{21}^{2} P_{1}+\right.$ $\left.2 h_{21} h_{22} \rho \sqrt{P_{1} P_{2}}+h_{22}^{2} P_{2}\right)$ and $D=2 h_{23}\left(h_{11} h_{21} P_{1}+\right.$ $\left.\left(h_{11} h_{22}+h_{12} h_{21}\right) \rho \sqrt{P_{1} P_{2}}+h_{12} h_{22} P_{2}\right)$. With $\alpha=\alpha_{e q}$, the RHS of (6b) can be attained, which constitutes the bound on 


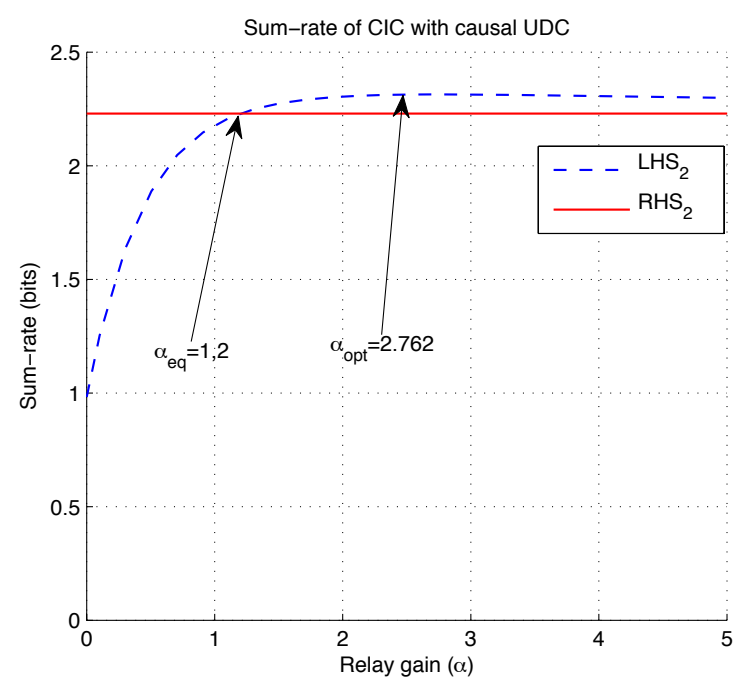

Fig. 2. The sum rates as functions of the relay amplification factor for $h_{11}=$ $h_{22}=h_{23}=h_{21}=1, h_{12}=4, \rho=0.5$ and $P_{1}=P_{2}=1$.

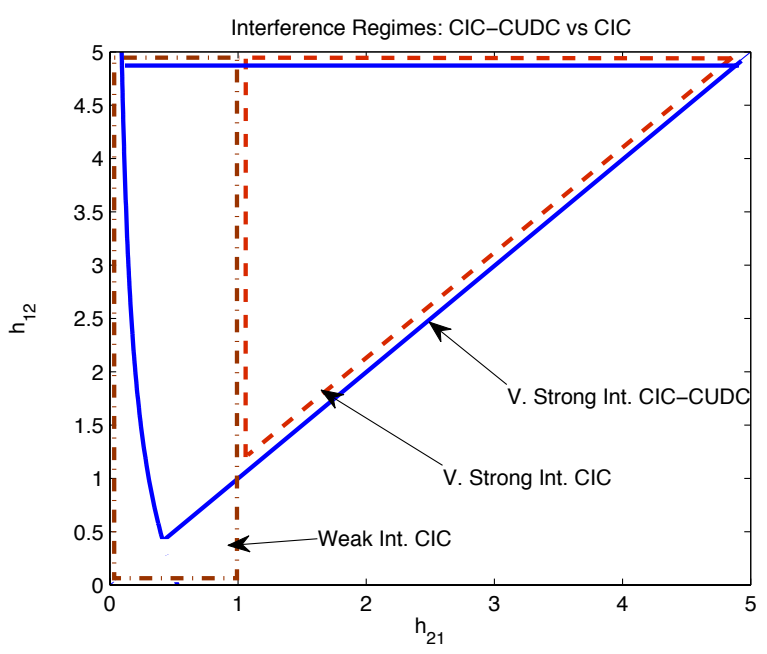

Fig. 3. Comparison of very strong interference regimes for the CIC and the CIC-CUDC for $h_{11}=h_{22}=h_{23}=P_{1}=P_{2}=1$.

the sum-rate in (4b). Fig. 3 shows the VSI regime for our model. This analysis assumes that $\alpha_{e q} y_{1} \leq P_{3}$. A relay with such characteristic is known as a potent relay [8]. Converse: We apply bounds (1a) and (1b) of Theorem 1 in the Gaussian model.

$$
\begin{aligned}
R_{1} \leq & I\left(X_{1} ; Y_{1}, X_{3} \mid X_{2}\right), \\
= & I\left(X_{1} ; Y_{1} \mid X_{2}\right)+I\left(X_{1} ; X_{3} \mid X_{2}, Y_{1}\right), \\
= & h\left(Y_{1} \mid X_{2}\right)-h\left(Y_{1} \mid X_{1}, X_{2}\right)+h\left(X_{3} \mid X_{2}, Y_{1}\right) \\
& \quad-h\left(X_{3} \mid X_{1}, X_{2}, Y_{1}\right), \\
& \stackrel{(a)}{=} h\left(h_{11} X_{1}+Z_{1} \mid X_{2}\right)-h\left(Z_{1}\right), \\
= & h\left(h_{11} X_{1}+Z_{1}, X_{2}\right)-h\left(X_{2}\right)-h\left(Z_{1}\right), \\
\leq & \mathcal{C}\left(h_{11}^{2}\left(1-\rho^{2}\right) P_{1}\right),
\end{aligned}
$$

and

$$
R_{1}+R_{2} \leq I\left(X_{1}, X_{2} ; Y_{1}, X_{3}\right)
$$

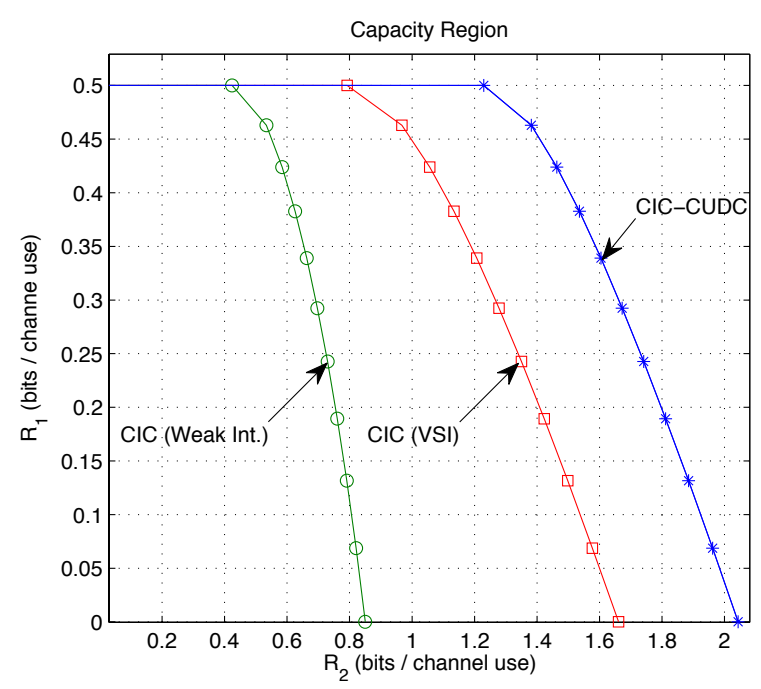

Fig. 4. Capacity region comparison between the CIC-CUDC in VSI against the CIC in weak and VSI. For the CIC, $h_{11}=h_{22}=P_{1}=P_{2}=1$, $h_{12}=3, h_{21}=2$ (VSI) and $h_{21}=0.5$ (Weak Int.). For the CIC-CUDC in VSI, $h_{11}=h_{22}=h_{23}=P_{1}=P_{2}=1, h_{12}=3$.

$$
\begin{aligned}
& \stackrel{(b)}{=} I\left(X_{1}, X_{2} ; Y_{1}\right), \\
& =h\left(Y_{1}\right)-h\left(Y_{1} \mid X_{1}, X_{2}\right) \\
& =h\left(h_{11} X_{1}+h_{12} X_{2}+Z_{1}\right)-h\left(Z_{1}\right) \\
& \leq \mathcal{C}\left(h_{11}^{2}+2 h_{11} h_{12} \rho \sqrt{P_{1} P_{2}}+h_{12}^{2} P_{2}\right),
\end{aligned}
$$

where $(a)$ and $(b)$ follow as $X_{3}=\alpha Y_{1}$.

It can be noted in Fig. 3 that the VSI region for the CIC-CUDC comprises the VSI region and part of the weak interference region of the CIC. This also indicates that only one and not two encoding schemes are necessary for much of the interference regimes for which capacity is known. We can also compare the capacity region of our model, the CICCUDC with that of the CIC in weak and VSI. Fig. 4 depicts this comparison. Note that the capacity region of the CICCUDC in VSI does not depend on the value of $h_{21}$ and as long the channel is in this regime the relay compensates any loss in the rate by making $\alpha=\alpha_{e q}$ for any variation of the channel gains. The variation of $\alpha_{e q}$ with respect to $h_{21}$ is depicted in Fig. 5.

\section{Conclusions}

We introduced the cognitive interference channel with causal unidirectional destination cooperation. We presented an outer bound for the discrete memoryless channel. For the Gaussian channel we showed that instantaneous AF relaying is sufficient to attain the capacity region in the very strong interference regime. We compared the very strong interference regime region of our model with those for the weak and VSI for the CIC. We observed that in our model the VSI region is enlarged allowing the utilization of only one encoding scheme rather than two, as in the CIC, for more values of the channel coefficients. We also compared the capacity regions and showed that the region of the CIC-CUDC does not depend 


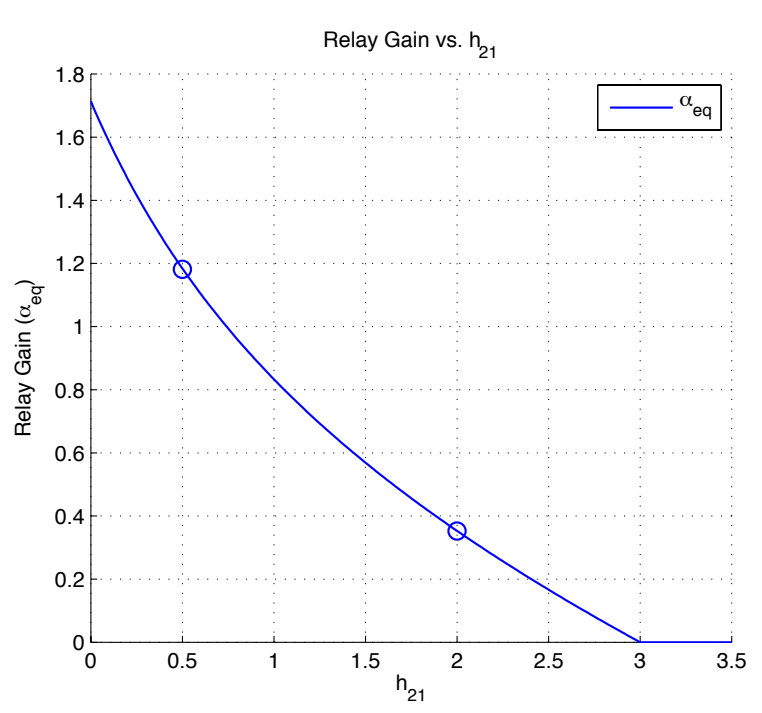

Fig. 5. The relay gain $\alpha_{e q}$ vs. the channel coefficient $h_{21}$. The values utilised for $h_{21}$ in Fig. 4 are marked with circles.

on $h_{21}$, whereas the capacity region of the CIC has a strong dependence on it.

\section{APPENDIX A}

\section{PROOF OF THEOREM 1}

From Fano's inequality [6]

$$
\begin{aligned}
n\left(R_{1}-\epsilon_{n}\right) & \leq I\left(M_{1} ; Y_{1}^{n} \mid M_{2}\right), \\
n\left(R_{1}+R_{2}-\epsilon_{n}\right) & \leq I\left(M_{1}, M_{2} ; Y_{1}^{n}\right), \\
n\left(R_{1}+R_{2}-\epsilon_{n}\right) & \leq I\left(M_{1}, M_{2} ; Y_{1}^{n}, Y_{2}^{n}\right) .
\end{aligned}
$$

Due to the lack of space we avoid the dependence on $\epsilon_{n}$ in the following. From (7)

$$
\begin{aligned}
n R_{1} & \leq \sum_{i=1}^{n} I\left(M_{1} ; Y_{1 i} \mid M_{2}, Y_{1}^{i-1}\right) \\
& =\sum_{i=1}^{n} H\left(M_{1} \mid M_{2}, Y_{1}^{i-1}\right)-H\left(M_{1} \mid M_{2}, Y_{1}^{i-1}, Y_{i}\right), \\
& =\sum_{i=1}^{n} H\left(M_{1} \mid M_{2}, Y_{1}^{i-1}\right)-H\left(M_{1} \mid M_{2}, Y_{1}^{i-1}, Y_{i}, X_{3 i}\right), \\
& =\sum_{i=1}^{n} I\left(M_{1} ; Y_{1 i}, X_{3 i} \mid M_{2}, Y_{1}^{i-1}\right), \\
& =\sum_{i=1}^{n} I\left(M_{1}, M_{2}, Y_{1}^{i-1}, X_{1 i} ; Y_{1 i}, X_{3 i} \mid X_{2 i}\right), \\
& \stackrel{(a)}{=} \sum_{i=1}^{n} I\left(X_{1 i} ; Y_{1 i}, X_{3 i} \mid X_{2 i}\right) .
\end{aligned}
$$

From (8), $n\left(R_{1}+R_{2}\right)$

$$
\begin{aligned}
& \leq \sum_{i=1}^{n} I\left(M_{1}, M_{2} ; Y_{1 i} \mid Y_{1}^{i-1}\right), \\
& =\sum_{i=1}^{n} H\left(M_{1}, M_{2} \mid Y_{1}^{i-1}\right)-H\left(M_{1}, M_{2} \mid Y_{1}^{i-1}, Y_{1 i}\right),
\end{aligned}
$$

$$
\begin{aligned}
& =\sum_{i=1}^{n} H\left(M_{1}, M_{2} \mid Y_{1}^{i-1}\right)-H\left(M_{1}, M_{2} \mid Y_{1}^{i-1}, Y_{1 i}, X_{3 i}\right) \\
& =\sum_{i=1}^{n} I\left(M_{1}, M_{2} ; Y_{1 i}, X_{3 i} \mid Y_{1}^{i-1}\right) \\
& \leq \sum_{i=1}^{n} I\left(M_{1}, M_{2}, Y_{1}^{i-1}, X_{1 i}, X_{2 i} ; Y_{1 i}, X_{3 i}\right) \\
& \stackrel{(b)}{\leq} \sum_{i=1}^{n} I\left(X_{1 i}, X_{2 i} ; Y_{1 i}, X_{3 i}\right)
\end{aligned}
$$

And from (9), $n\left(R_{1}+R_{2}\right)$

$$
\begin{aligned}
& \leq \sum_{i=1}^{n} I\left(M_{1}, M_{2} ; Y_{1 i}, Y_{2 i} \mid Y_{1}^{i-1}, Y_{2}^{i-1}\right), \\
& =\sum_{i=1}^{n} I\left(M_{1}, M_{2} ; Y_{1 i} \mid Y_{1}^{i-1}, Y_{2}^{i-1}\right) \\
& \quad+\sum_{i=1}^{n} I\left(M_{1}, M_{2} ; Y_{2 i} \mid Y_{1}^{i-1}, Y_{1 i}, Y_{2}^{i-1}\right), \\
& =\sum_{i=1}^{n} I\left(M_{1}, M_{2}, X_{1 i}, X_{2 i} ; Y_{1 i} \mid Y_{1}^{i-1}, Y_{2}^{i-1}\right) \\
& \quad+\sum_{i=1}^{n} I\left(M_{1}, M_{2}, X_{1 i}, X_{2 i} ; Y_{2 i} \mid Y_{1}^{i-1}, Y_{1 i}, Y_{2}^{i-1}, X_{3 i}\right), \\
& \stackrel{(c)}{=} \sum_{i=1}^{n} I\left(X_{1 i}, X_{2 i} ; Y_{1 i}\right)+\sum_{i=1}^{n} I\left(X_{1 i}, X_{2 i} ; Y_{2 i} \mid Y_{1 i}, X_{3 i}\right),
\end{aligned}
$$

where $(a),(b)$ and $(c)$ follow from the memoryless property of the channel. The rest of the proof is customary and follows by introducing a time-sharing random variable $Q$, uniformly distributed in $[1: n]$ and independent of $\left(M_{1}, M_{2}, X_{k}^{n}, X_{3}^{n}, Y_{k}^{n}\right)$ for $k=1,2$ and defining $X_{k}=X_{k Q}$ and $Y_{k}=Y_{k Q}$.

\section{ACKNOWLEDGEMENT}

This work is supported by the India-UK Advanced Technology Centre of Excellence in Next Generation Networks (IUATC) project jointly funded by the Department of Science and Technology (DST), Government of India and by the Engineering and Physical Sciences Research Council (EPSRC), UK.

\section{REFERENCES}

[1] N. Devroye, P. Mitran, and V. Tarokh, "Achievable Rates in Cognitive Radio Channels," IEEE Trans. Inf. Theory, vol. 52, no. 5, pp. 1813-1827, May 2006.

[2] A. Goldsmith, S. A. Jafar, I. Marić, and S. Srinivasa, "Breaking Spectrum Gridlock With Cognitive Radios: An Information Theoretic Perspective," Proceedings of the IEEE, vol. 97, no. 5, pp. 894-914, Sep. 2009.

[3] W. Wu, S. Vishwanath, and A. Arapostathis, "Capacity of a Class of Cognitive Radio Channels: Interference Channels With Degraded Message Sets," IEEE Trans. Inf. Theory, vol. 53, no. 11, pp. 4391-4399, Nov. 2007.

[4] H.-Y. Chu and H.-J. Su, "On the capacity region of the cognitive interference channel with unidirectional destination cooperation," in Proc. IEEE Int. Symp. Information Theory, Saint Petersburg, Russia, Jul. 2011, pp. 2408-2412.

[5] M. Kazemi and A. Vosoughi, "On the Capacity Region of the Partially Cooperative Relay Cognitive Interference Channel," in Proc. IEEE Int. Symp. Information Theory, Istambul, Turkey, Jul. 2013, pp. 2424-2427.

[6] A. El Gamal and Y.-H. Kim, Network Information Theory. New York: Cambridge University Press, 2011. 
[7] I. Marić, R. D. Yates, and G. Kramer, "Capacity of Interference Channels With Partial Transmitter Cooperation," IEEE Trans. Inf. Theory, vol. 53, no. 10, pp. 3536-3548, 2007.

[8] H. Chang, S.-y. Chung, and S. Kim, "Interference Channel With a Causal Relay Under Strong and Very Strong Interference," IEEE Trans. Inf. Theory, vol. 60, no. 2, pp. 859-865, 2014. 